\title{
Engineered collagen hydrogels for the sustained release of biomolecules and imaging agents: promoting the growth of human gingival cells
}

This article was published in the following Dove Press journal:

International Journal of Nanomedicine

II November 2014

Number of times this article has been viewed

\author{
Jonghoon Choi ${ }^{1,2}$ \\ Hoyoung Park ${ }^{3}$ \\ Taeho Kim ${ }^{4,5}$ \\ Yoon Jeong ${ }^{1,2}$ \\ Myoung Hwan $\mathrm{Oh}^{4,5}$ \\ Taeghwan Hyeon ${ }^{4,5}$ \\ Assaf A Gilad ${ }^{6,7}$ \\ Kwan Hyi Lee ${ }^{3}$
}

'Department of Bionanotechnology, Hanyang University, Seoul Campus, Seoul, ${ }^{2}$ Department of Bionanoengineering, Hanyang University, ERICA Campus, Ansan, ${ }^{3}$ Center for Biomaterials, Biomedical Research Institute, Korea Institute of Science and Technology, Seoul, ${ }^{4}$ Center for Nanoparticle Research, Institute for Basic Science, Seoul, ${ }^{5}$ School of Chemical and Biological Engineering, Seoul National University, Seoul, Korea; ${ }^{6}$ Department of Radiology and Radiological Health, Johns Hopkins University School of Medicine, Baltimore, MD, USA; ${ }^{7}$ Institute for Cell Engineering, Baltimore, MD, USA

\section{Correspondence: Kwan Hyi Lee Center for Biomaterials, Biomedical Research Institute, Korea Institute of Science and Technology, Seoul, Korea Email kwanhyi@kist.re.kr}

Jonghoon Choi

Department of Bionano Technology, Hanyang University, Seoul Campus, Seoul, Korea

Email jonghchoi@hanyang.ac.kr

\begin{abstract}
We present here the in vitro release profiles of either fluorescently labeled biomolecules or computed tomography contrast nanoagents from engineered collagen hydrogels under physiological conditions. The collagen constructs were designed as potential biocompatible inserts into wounded human gingiva. The collagen hydrogels were fabricated under a variety of conditions in order to optimize the release profile of biomolecules and nanoparticles for the desired duration and amount. The collagen constructs containing biomolecules/nanoconstructs were incubated under physiological conditions (ie, $37^{\circ} \mathrm{C}$ and $5 \% \mathrm{CO}_{2}$ ) for 24 hours, and the release profile was tuned from $20 \%$ to $70 \%$ of initially loaded materials by varying the gelation conditions of the collagen constructs. The amounts of released biomolecules and nanoparticles were quantified respectively by measuring the intensity of fluorescence and X-ray scattering. The collagen hydrogel we fabricated may serve as an efficient platform for the controlled release of biomolecules and imaging agents in human gingiva to facilitate the regeneration of oral tissues.
\end{abstract}

Keywords: engineered collagen hydrogels, biomolecules, imaging agents, sustained release, human gingival cells, growth

\section{Introduction}

A sufficient supply of biomolecules (eg, growth factors) is critical for gingival fibroblasts to be able to proliferate in wounded gum. ${ }^{1,2}$ Without a supply of blood and responsible biomolecules, only a small number of cells may survive. Placement of wound-healing hydrogels at dental implant sites for rapid recruitment of vascular capillaries and sufficient delivery of biomolecules is a promising way to achieve less painful and more efficient healing of wounded gum. ${ }^{3,4}$

Growth factors such as vascular endothelial growth factor and basic fibroblast growth factor stimulate endothelial cells to migrate from the surrounding tissue and proliferate. ${ }^{5}$ However, simple injection of these biomolecules at the wound site fails to stimulate this process efficiently because the biomolecules easily diffuse away from the injection site. Controlled protein release from biopolymer scaffolds/hydrogels has been investigated in order to enhance the effects of delivery of growth factors. ${ }^{6,7}$

Collagen is one of the major extracellular matrix components in mammals. It is a hydrogel composed of structural proteins that provides mechanical integrity to a variety of tissues and organs and stores several biomolecules. ${ }^{8}$ Collagen substrates have been shown to promote the proliferation, migration, and differentiation of human gingival fibroblasts. ${ }^{9}$ Numerous studies have sought to improve and reproduce the growth and differentiation of fibroblasts and epidermal cells by using collagenous materials 
as support. ${ }^{10-14}$ Three-dimensional culture methods using collagen gels successfully adhered to the collagen fibers and the cytoplasmic processes of the cultured cells to the fibers of the carrier grids without contracting or detaching from the supporting structures. Collagen hydrogels are, therefore, an ideal substrate for use in damaged gingival tissue for faster healing of the wound site.

The successful control of the release of biomolecules has been demonstrated by controlling the quality of the hydrogel. ${ }^{15}$ The release profile of drugs and/or biomolecules from engineered collagen constructs depends primarily on how hydrogels are prepared. The diffusion of biomolecules, nanoparticles, and drugs can be regulated by fabrication technique, crosslinking density, nature of the cross-linker, and/or quaternary structures. ${ }^{8,16,17}$ Any conformational changes of hydrogels and binding affinities of the collagen networks may influence transportation phenomena of fluid, which may affect the diffusion kinetics and entrapment efficacy of the drugs. ${ }^{18,19}$

To assess its potential in in vivo applications, molecular imaging modalities, eg, fluorescence, computed tomography (CT), and magnetic resonance imaging (MRI), are often adapted to quantify the amount of biomolecules/nanoparticles released from engineered scaffolds or hydrogels used as drug carriers. ${ }^{20}$ MRI can quantify the amount of released drug molecules attached to iron oxide nanoparticles using the magnetic properties of nanoparticles. ${ }^{21} \mathrm{CT}$ has also been used to image gold nanoparticles to measure the distribution and trafficking of drug molecules conjugated with nanoparticles. ${ }^{22}$

In this study, we engineered a collagen hydrogel that is implantable into human gingival tissue, and examined the in vitro release profiles of fluorescently labeled or CT contrast biomolecules and nanoparticles from the hydrogel. Coculture of deliberately damaged gingival cell clusters with the engineered collagen hydrogels facilitated the recovery of the cell clusters. Proliferation of gingival cells incubated with transforming growth factor (TGF)- $\beta 1$-releasing hydrogels was examined in in vitro time-lapse studies.

\section{Materials and methods Materials}

Rat tails were generously donated by Dr Nakwon Choi at the Korea Institute of Science and Technology. Primary human gingival fibroblasts were obtained from donated teeth collected at the Soon Chun Hyang Hospital in Seoul, Republic of Korea. Reagents were purchased from the following companies: antibiotic-antimycotic, 100× (Gibco, Grand Island, NY, USA; catalog number 15240-062), MycoGONE mycoplasma antibiotic cocktail, 50× (Genlantis, San Diego, CA, USA; catalog number A200100), fetal bovine serum, (Welgene, Daegu, Republic of Korea; catalog number S001-07), Dulbecco's phosphate-buffered saline (PBS; Welgene, catalog number LB 001-02), Dulbecco's Modified Eagle's Medium (DMEM), high glucose, pyruvate (Gibco, catalog number 11995-073), AuroVist ${ }^{\mathrm{TM}} 15 \mathrm{~nm}$ gold nanoparticle X-ray contrast agent (Nanoprobe, Yaphank, NY, USA; catalog number 1115), fluorescein isothiocyanate (FITC)-dextran 500 kDa (Sigma-Aldrich, St Louis, MO, USA; catalog number FD500S-100MG), recombinant human TGF- $\beta 1$ (R\&D Systems, Minneapolis, MN, USA; catalog number 240-B-002), Nanodot HE-100-680 nm (QD Solutions, Austin, TX, USA: catalog number HE-100-620 nm), anti-alpha smooth muscle actin antibody (Abcam, Cambridge, MA, USA; catalog number ab8211), 1-myristoyl-2-hydroxy-sn-glycero3-phosphocholine (Avanti Polar Lipids, Alabaster, AL, USA; catalog number 855575C), 1,2-distearoyl-sn-glycero3-phosphoethanolamine-N-(methoxy polyethylene glycol)2000 (Avanti Polar Lipids; catalog number 880120C), and 1,2-dioleoyl-sn-glycero-3-N-(5-amino-1-carboxypentyl) iminodiacetic acid succinyl nickel salt (Avanti Polar Lipids; catalog number 790404C). All reagents were of analytical grade and used as received.

\section{Preparation of collagen hydrogel}

Type I collagen was extracted from rat tail tendons and processed in acetic acid solution to obtain sterile soluble collagen. ${ }^{23}$ Lyophilized collagen $(93 \mathrm{mg}$ ) was dissolved in $6.2 \mathrm{~mL}$ of acetic acid to make $1.5 \%(\mathrm{w} / \mathrm{v})$ collagen stock solution. An example preparation is as follows: $780 \mu \mathrm{L}$ of stock collagen was mixed with $300 \mu \mathrm{L}$ of DMEM. Subsequently, $58.5 \mu \mathrm{L}$ of $100 \mu \mathrm{M}$ FITC-dextran (molecular weight $500 \mathrm{kDa}$ ) was added to the collagen solution for fluorescence imaging. An appropriate amount of $1 \mathrm{M} \mathrm{NaOH}$ solution was then applied to neutralize the collagen mixture at a $\mathrm{pH}$ of $\sim 7$. To make a cone-shaped collagen sample, we first filled the prepared polydimethylsiloxane mold with the collagen mixture. The mixture was then allowed to undergo gelation at $37^{\circ} \mathrm{C}$ for 1 hour, leading to a porous collagen structure. For our transient release experiment, we tested a total of six collagen construct samples.

\section{Synthesis of ferritin nanoparticles}

To verify the effective pore sizes of the collagen constructs, we carried out an additional release experiment using ferritin nanoparticles. We engineered the human ferritin gene structure to allow for expression of protein $\mathrm{G}$ and six 
histidines on the surface of ferritin. Ni-Nitrilotriacetate metal chelator is well known for binding histidine protein. Synthesized ferritin particles were incubated with antialpha smooth muscle actin antibodies at a 1:1 molar ratio to maximize the area of functionalized surface. Using Ni-functionalized quantum dots, the ferritin nanostructure was decorated with water-soluble quantum dots at a ratio of $1: 7 .{ }^{24,25}$

\section{Synthesis of $\mathrm{TaO}$ nanoparticles}

Tantalum oxide $(\mathrm{TaO})$ nanoparticles were synthesized by microemulsion methods. ${ }^{26}$ First, a microemulsion was prepared by mixing $4.6 \mathrm{~g}$ of Igepal CO-520 (Sigma-Aldrich), $0.5 \mathrm{~mL}$ of ethanol 99.5\% (Samchun Pure Chemical Co Ltd, Pyeongtaek, Republic of Korea), $0.5 \mathrm{~mL}$ of $\mathrm{NaOH}$ aqueous solution $(75 \mathrm{mM})$, and $40 \mathrm{~mL}$ of cyclohexane $99.5 \%$ (Samchun Pure Chemical Co Ltd), in a $100 \mathrm{~mL}$ roundbottomed flask. After adding $0.1 \mathrm{~mL}$ of tantalum (V) ethoxide (0.3 mmol, 99.8\%; Strem Chemicals Inc., Newburyport, MA, USA) to the microemulsion at room temperature, $\mathrm{TaO}$ nanoparticles were synthesized within 5 minutes. We added $0.5 \mathrm{~mL}$ of 2-methoxy (polyethyleneoxy) propyltrimethoxysilane (PEG-silane, 596-725 Da; Gelest, Greenville, SC, USA) for pegylation of the $\mathrm{TaO}$ nanoparticles. Rhodamine-B-isothiocyanate (RITC) was further functionalized on the surface of $\mathrm{TaO}$ nanoparticles by adding $10 \mu \mathrm{L}$ of RITC-aminopropyltriethoxysilane (APTES, Sigma-Aldrich) solution to the reaction mixtures. RITC-APTES stock solution was prepared in advance by reaction of $110 \mu \mathrm{L}$ of APTES with $50 \mathrm{mg}$ of RITC in $3.75 \mathrm{~mL}$ of ethanol for 24 hours. The mixture was then stirred for 24 hours and the as-synthesized nanoparticles were washed with a mixed solution of $1: 1(\mathrm{v} / \mathrm{v})$ ether/nhexane and dispersed in ethanol. Finally, to conjugate PEG onto residual amine groups on the surfaces of the functionalized $\mathrm{TaO}$ nanoparticles, $20 \mathrm{mg}$ of methoxypoly(ethylene glycol) succinimidyl glutarate (molecular weight 2000, Sunbio Inc, Gyeonggi-do, Republic of Korea) dissolved in $10 \mathrm{~mL}$ of ethanol was mixed and stirred overnight at $30^{\circ} \mathrm{C}$. After washing several times with deionized water, the final product, PEG-RITC-TaO, was dispersed in PBS.

\section{Characterization of nanoparticles}

Transmission electron microscopy analysis was conducted with a JEM-2100F transmission electron microscope (JEOL Ltd, Tokyo, Japan) operating at $200 \mathrm{kV}$. Samples were prepared by putting a drop of particle dispersions onto a carbon-coated copper grid.

\section{Loading of biomolecules/nanoparticles into hydrogels}

Lyophilized TGF- $\beta 1$ (R\&D Systems) was dissolved in culture medium, aliquoted, and stored at $-70^{\circ} \mathrm{C}$. To assess the effects of growth factor, cells were cultured in $0.1 \%$ fetal bovine serum medium containing $0-100 \mathrm{ng} / \mathrm{mL}$ of TGF- $\beta 1$. Collagen hydrogels were loaded with either TGF- $\beta 1$ or other nanoparticles (eg, gold, $\mathrm{TaO}$, dextran, or ferritin nanoparticles), and the resulting swollen hydrogels were kept at $4^{\circ} \mathrm{C}$ for 2 hours before being transferred into buffer solution. Typical impregnations of biomolecules into collagen hydrogels were carried out with PBS containing target molecules. The controls, ie, empty collagen hydrogels, were prepared under the same protocol. All hydrogels prepared by this method were similar in appearance, regardless of hydrogel type and labeling.

\section{Release profiling of biomolecules/ nanoparticles}

In vitro degradation of collagen hydrogels was evaluated in terms of fluorescence emitted by either dye molecules attached to the released dextran particles or quantum dots conjugated to the ferritin nanoparticles. To calculate the amounts of FITC-dextran released from the collagen cones, all samples were submerged in $2.5 \mathrm{~mL}$ of PBS solution. Samples containing the FITC-dextran, diffused from the collagen cones, were taken at 1,2 , $3,6,12$, and 24 hours immediately after submergence in PBS. We quantified the amount of released FITCdextran by measuring the fluorescence intensity of the PBS samples. We also measured the amount of TGF- $\beta 1$ released in the PBS samples using a Quantikine human TGF- $\beta 1$ immunoassay kit ( $R \& D$ systems). In addition to these quantifications, $\mathrm{CT}$ measurements of $\mathrm{TaO}$ nanoparticles released from the hydrogels were translated into the amounts of particles in solution. Micro-CT imaging was performed on a Caliper IVIS Spectrum CT system. Samples were positioned on a rotating platform placed in front of a flat panel detector and an X-ray tube. A high-resolution detector with a $150 \mu \mathrm{m}$ voxel size and a resolution of 425 was used over an active area of $12 \times 12 \times 13 \mathrm{~cm}$ (length, width, height). We acquired 720 projections for reconstructing tomograms. The hardware used allowed averaging of the values of a $4 \times 4$ array of pixels, which in turn increased the signal to noise ratio. The total experimental time took $\sim 140$ seconds per trial, with an average dose of about $52.8 \mathrm{mGv}$. 


\section{Human gingival cell culture}

Human gingival tissues were obtained from donated extracted wisdom teeth at Soon-Chun-Hyang Hospital, Seoul, Republic of Korea. The study was approved by the institutional review boards of Soon-Chun-Hyang Hospital and Korea Institute of Science and Technology, Seoul, Republic of Korea. Written informed consent was obtained from all participants. None of the three male subjects studied had any sign of malignant tumors or other diseases.

The obtained tissues were kept in cold DMEM with 10\% fetal bovine serum and a cocktail of antibiotics and antimycotics. Next, the tissues were minced with a blade and spun down at $4^{\circ} \mathrm{C}$ and $200 \times g$ for $5-10$ minutes to separate and wash the pellets. Resuspended tissue explants were laid onto a $60 \mathrm{~mm}$ dish and kept in an incubator. Every 3 days during culture, fibroblasts grown from tissue explants were washed and continued under passage using $0.2 \%$ trypsin. Proliferation tests and cell damage recovery experiments were performed when the fibroblasts reached passages 3-10.

\section{Recovery of damaged gingival cell clusters} Human gingival cells grown to confluence were scratched with a cell scraper (Nunc ${ }^{\circledR}$, Sigma-Aldrich). The resulting debris was removed by gentle washing with medium. The initial boundaries and areas of scratched surfaces were recorded and the cells were placed in an incubator. Cells were maintained for up to 24 hours in standard culture medium. Images of the healing wound were acquired by optical microscopy and analyzed using image analysis software (ImageJ).

\section{Proliferation assay}

The cell growth assay was performed using a method reported elsewhere. ${ }^{27}$ Briefly, fibroblasts $(25,000$ cells) in $2 \mathrm{~mL}$ of DMEM $10 \%$ were allowed to settle in a well of a 24-well culture plate (Corning Corporation, Cambridge, MA, USA) for 2 hours. After slight washing with PBS, the medium was replaced by DMEM containing $1.0 \%$ fetal bovine serum (DMEM 1\%) for 12 hours. Cells treated with TGF- $\beta 1$, hydrogel carrying TGF- $\beta 1$, and PBS (control) were harvested using $0.2 \%$ trypsin in PBS, and stained with Calcein-AM for live cell counting at an emission wavelength of $540 \mathrm{~nm}$ in a fluorescence microscope. Fluorescence intensities relating to the number of cells were recorded for analysis. All experiments were performed in triplicate.

\section{Statistical analysis}

Chi-square tests with Yate's correction and one-tailed Mann-Whitney $U$-tests were performed using GraphPad
Prism (version 5.0b for Mac OS X, GraphPad Software, San Diego, CA, USA).

\section{Results and discussion}

Figure 1 is an overview of our approach to the use of collagen hydrogel for implantation in damaged gingiva. Biomolecules such as TGF- $\beta 1$ released from the hydrogel are absorbed into neighboring tissue and assist in the recruitment and proliferation of gingival fibroblasts. ${ }^{4}$ Nanoparticles used for optical or CT contrast agents are also released from the hydrogel and were used to assess the release dynamics. To obtain a collagen hydrogel consisting of biomolecules and nanoparticles, simple mixtures of collagen extracts from rat tails and target molecules was prepared (Figure 2A). Target molecules listed in the table (Figure 2B) were characterized by their mean diameters, shapes, surface charges, and functions. The small (15 $\mathrm{nm}$ for gold and $30 \mathrm{~nm}$ for $\mathrm{TaO}$ ) nanoparticles were examined regarding the rate of release using contrast in X-ray imaging. $\mathrm{TaO}$ nanoparticles carried RITC on their surfaces, allowing for the use of dual modality imaging (CT and fluorescence). The largest $(100 \mathrm{~nm})$ fluorescent particles (ie, FITC-conjugated dextran particles) were examined for their release kinetics by measuring the fluorescent intensity of the supernatants containing released particles. Other samples (ie, TGF- $\beta 1$ molecules or ferritin nanoclusters) had an average mean diameter of $50 \mathrm{~nm}$ and were tested for their release from engineered collagen hydrogels.

The unique fluorescent signatures of dextran, $\mathrm{TaO}$, and ferritin particles led to visible coloration of the collagen hydrogels (Figure 3A). The loading efficiencies of each particle in the hydrogels were $2.4 \mathrm{mg} / \mathrm{mL}$ for dextran, $2 \mathrm{nM}$ for $\mathrm{TaO}$ nanoparticles, and $5 \mathrm{nM}$ for ferritin nanoclusters. The $\mathrm{pH}$ level during collagen hydrogel synthesis plays an important role in pore size optimization to maximize the loading of target particles. After swelling, the matrix structure of collagen opens up aqueous channels wide enough to let nanoparticles pass through easily, thus expediting the diffusion-dominated release of nanoparticles from collagen hydrogels. The release kinetics of the included particles depend on the extent of crosslinking, which may affect the swelling rate of collagen hydrogels. ${ }^{28}$

The release profile of each fluorescent particle from collagen hydrogels was measured in terms of fluorescence (Figure 3B). The standard curve was constructed by measuring the fluorescence intensities of standards (samples with predetermined concentrations). Fluorescence intensities measured for the experimental groups were back-calculated 


\section{A}

Concept of drug release and the implantable collagen hydrogel

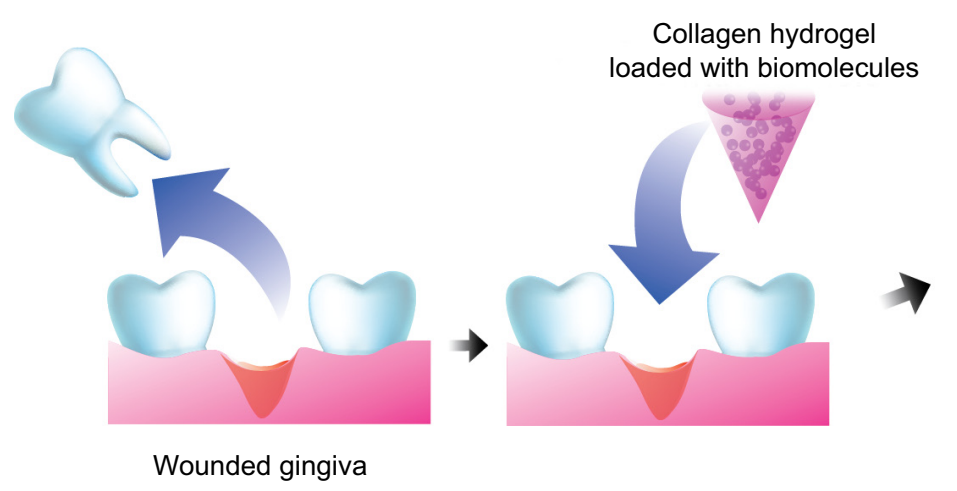

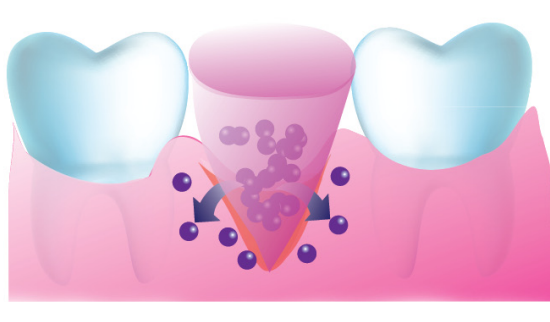

Collagen releasing biomolecules promoting cell and tissue recovery

\section{B} In vitro cell responses

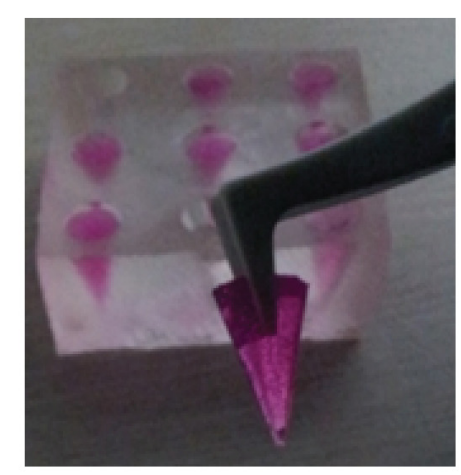

Collagen hydrogel
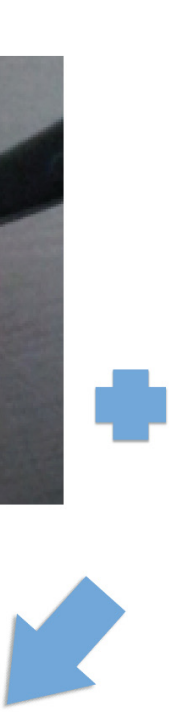

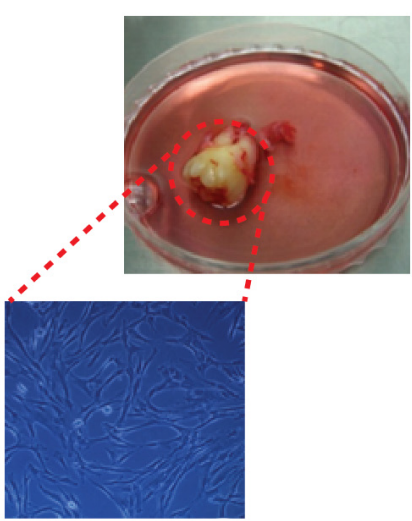

Gingival fibroblasts

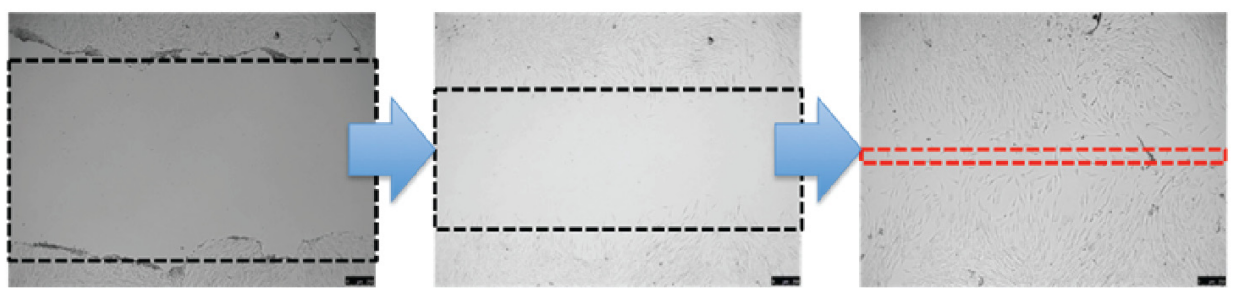

Facilitated cell recovery

Figure I Schematic representation of drug release and the implantable collagen hydrogel (A). The collagen hydrogel inserted into the wounded gingiva may release biomolecules that facilitate the recovery of damaged tissues. In vitro testing of the engineered hydrogel for its potential in supporting the fast proliferation of gingival cells (B).

to obtain unknown concentrations using the standard curve. As described in Figure 3B, dextran particles exhibited the most rapid release due to their largest diameters $(100 \mathrm{~nm}$ on average). In contrast, ferritin nanoclusters were the slowest sample to be released from the hydrogel, probably owing to their smaller size ( $50 \mathrm{~nm}$ in diameter, and more interaction with pores in the collagen matrix) and stronger electrostatic interactions between ions in the protein and collagen matrix. It is suspected that this electrostatic interaction is also responsible for the slower release of ferritin nanoclusters than $\mathrm{TaO}$ nanoparticles despite the larger size of ferritin nanoclusters (average diameters were $50 \mathrm{~nm}$ for the ferritin nanoclusters and $30 \mathrm{~nm}$ for the $\mathrm{TaO}$ nanoparticles).

Since collagen is a polyampholyte, a polyelectrolyte consisting of charged, hydrophobic amino acid side chains, the binding affinities of proteins or particles loaded into the matrix may be critically dependent on the collagen's porosity and/or electrostatic charge interactions. The primary 
A

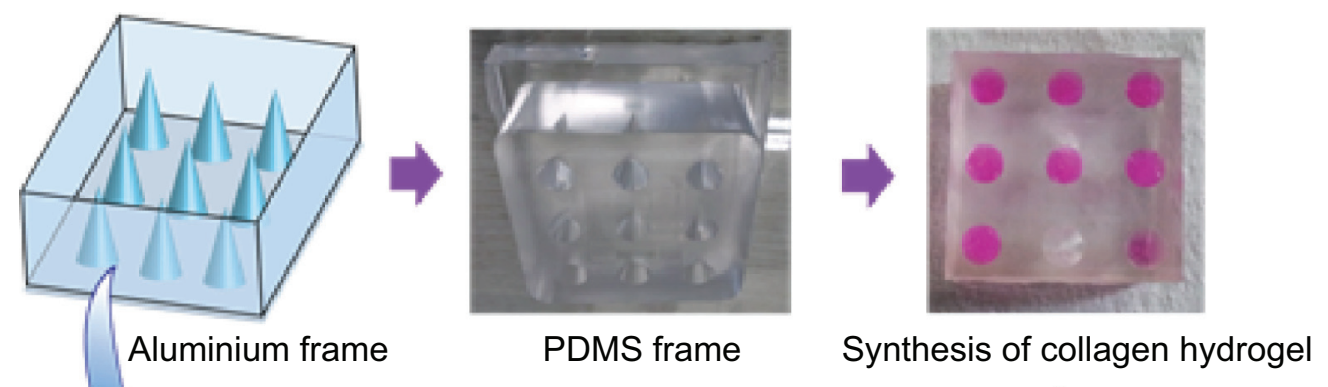

B

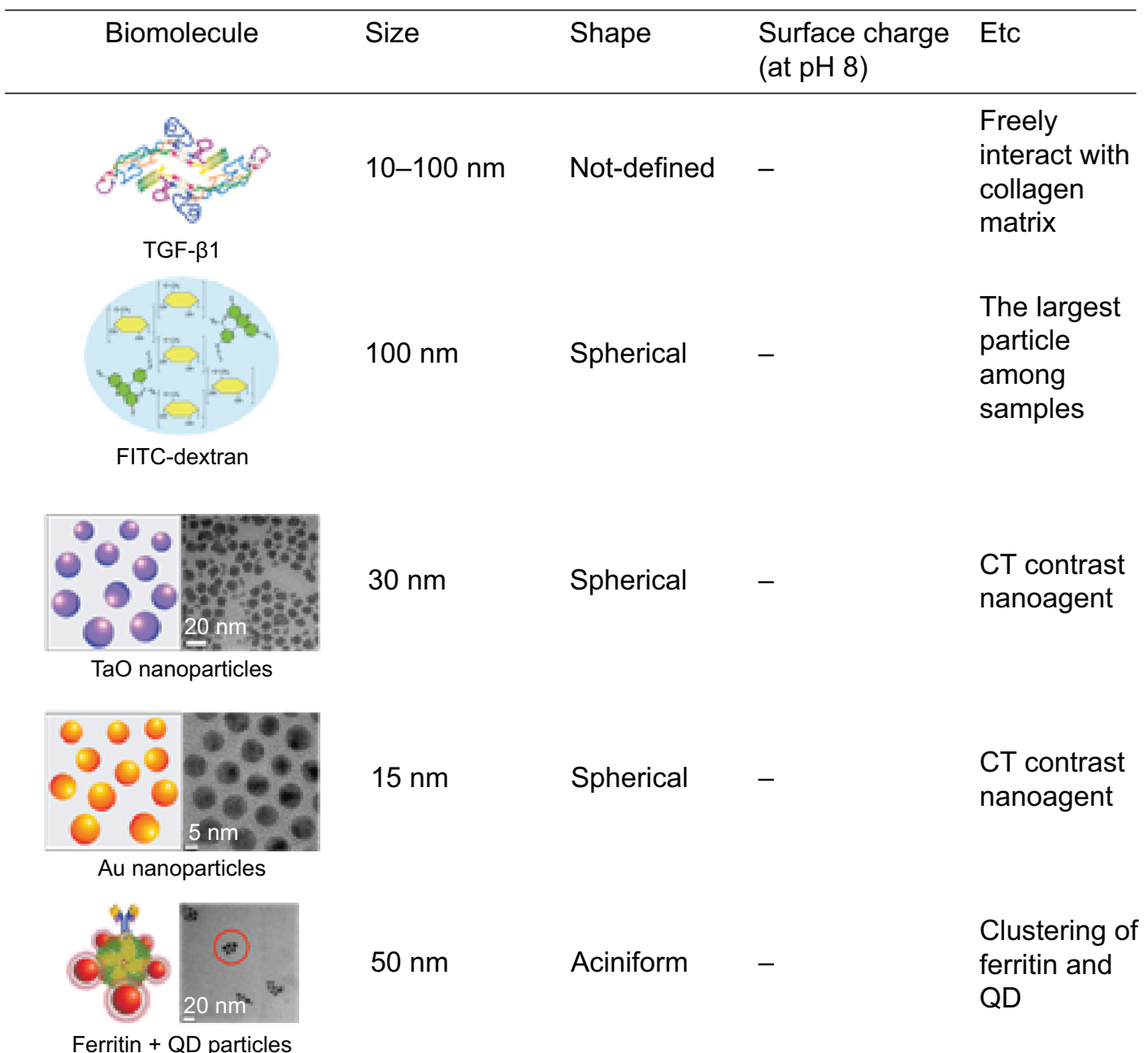

Figure 2 Collagen hydrogel preparation and constituents loaded in the hydrogel.

Notes: The cone-shaped hydrogel is fabricated in a PDMS frame by thermal curing (A). The characteristics of biomolecules and nanoparticles loaded in the hydrogel system are shown (B).

Abbreviations: TGF- $\beta$ I, transforming growth factor-beta I; PMDS, polydimethylsiloxane mold; FITC, fluorescein isothiocyanate; QD, quantum dots; CT, computed tomography; $\mathrm{TaO}$, tantalum oxide. 
A

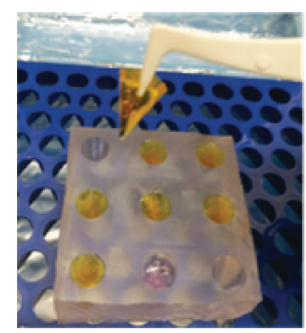

Load particles into the collagen

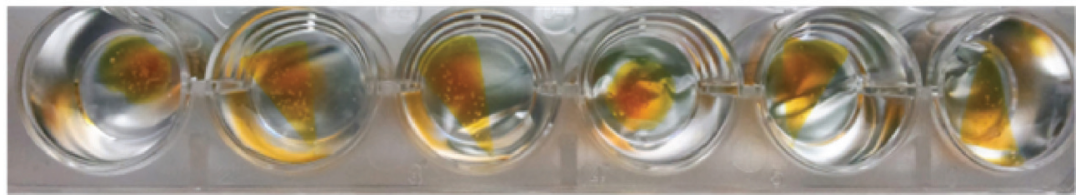

1

2

3

6

Submerge

hydrogel in PBS
Measure the fluorescence intensity
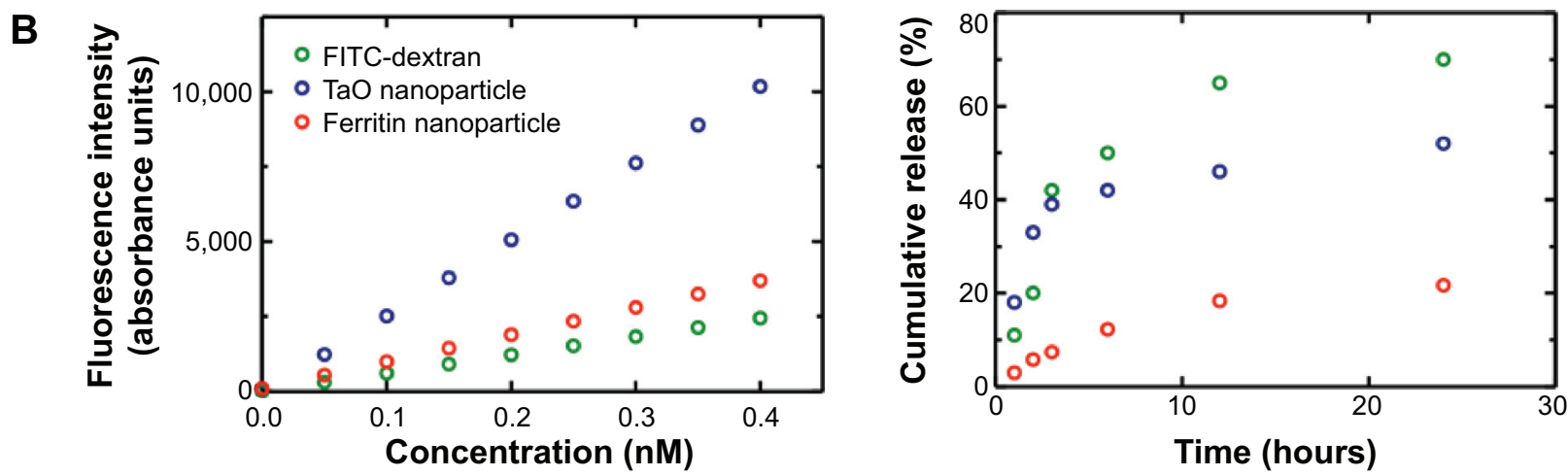

Figure 3 Nanoparticles packed in hydrogel displayed delayed release under physiological conditions.

Notes: Hydrogels encompassing nanoparticles are kept in PBS for predetermined periods of time (A). Fluorescence measured in wells containing hydrogels results from supernatants carrying released particles. The cumulative release of particles is calculated based on a standard curve constructed by measuring fluorescence intensities of different concentrations of standard (B).

Abbreviations: PBS, phosphate-buffered saline; FITC, fluorescein isothiocyanate; $\mathrm{TaO}$, tantalum oxide.

binding mechanisms for collagen are either electrostatic or hydrophobic in nature, and the strength of these interactions would be important to determine the kinetics of target release with different sizes. ${ }^{19,29,30}$ Indepth studies of the mechanisms of interaction between protein particles and collagen pores are necessary to clarify the release profile.

The application of nanoparticulate imaging probes to $\mathrm{X}$-ray CT imaging could have a significant impact on health care, owing to the ubiquitous availability of $\mathrm{CT}$ in the clinical setting as well as the increasing use and development of micro-CT and other advanced CT imaging systems. ${ }^{22}$ The iodinated molecules traditionally used as $\mathrm{CT}$ contrast agents in the clinic have relatively short circulation times in vivo, which significantly restricts the applications of this technique in target-specific imaging and angiography. ${ }^{31}$ In addition, the use of these agents can result in adverse effects for patients. Therefore, the development of a new generation of CT contrast agents using nanoparticles composed of other elements with higher X-ray attenuation would mark another breakthrough in CT imaging. ${ }^{22,26,31,32}$ The design of new agents should also be guided by other criteria, such as low toxicity. In our study, we evaluated the release dynamics of our collagen hydrogel system with two different nanoparticulate imaging probes (ie, gold or $\mathrm{TaO}$ nanoparticles) using particle $\mathrm{CT}$ contrast (Figure 4A). X-ray fluoroscopy (Figure 4B) shows that an unknown concentration of nanoparticles can be calculated using X-ray absorption measurements. Figure 4C shows an experimental calibration curve of X-ray attenuation as a function of nanoparticle concentration and determination of unknown concentration using the calibration curve. The cumulative release of $\mathrm{TaO}$ nanoparticles is initially more rapid (less than 1 hour post incubation) than that of gold nanoparticles from the hydrogel, while maintaining a similar subsequent rate of release (Figure 4). Because the average diameter of the $\mathrm{TaO}$ nanoparticles $(30 \mathrm{~nm})$ is larger than that of the gold particles $(15 \mathrm{~nm})$, TaO nanoparticles were secreted more rapidly in the earlier stages until the excretion rates stabilized after 1 hour. In addition to the size effect, previous studies have shown the importance of porosity and/or surface charge for the efficient permeation occurred through the "bulk-like" water regions or by a "pore"-type mechanism. ${ }^{16}$ Also, increasing the charge density resulted in increased binding interactions, thereby retarding the release rates.

Collagen is not degraded by simple hydrolysis but by proteolysis. Therefore, the release of substances mixed within the collagen matrix is contingent on the speed of collagen 
A

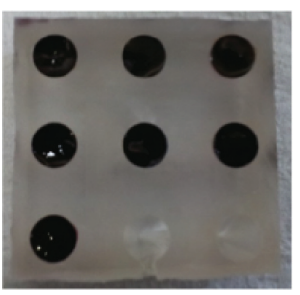

Load particles into the collagen

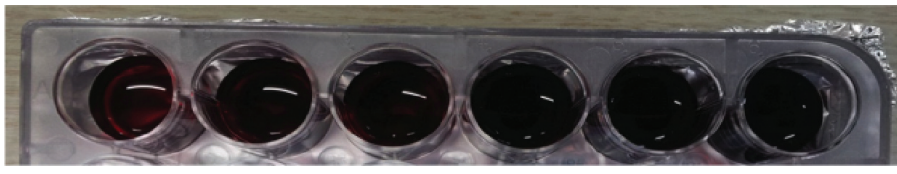

1

2

3

6

12

24

Hours

Submerge hydrogel in PBS
Measure the CT intensity
B
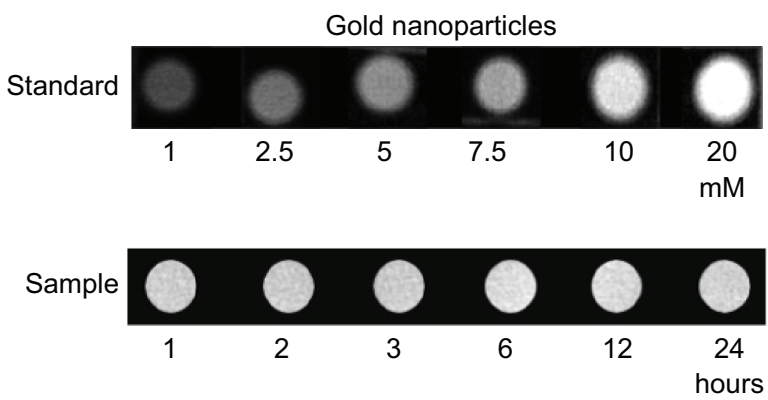

TaO nanoparticles
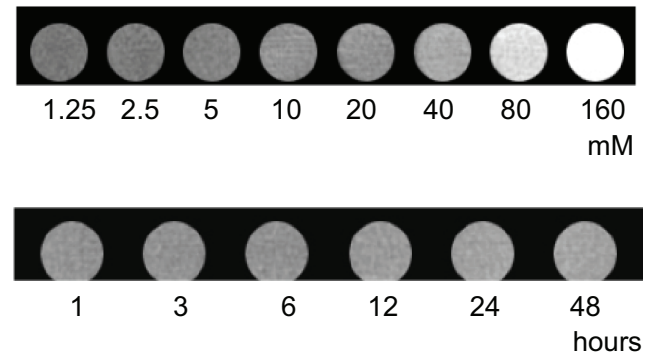
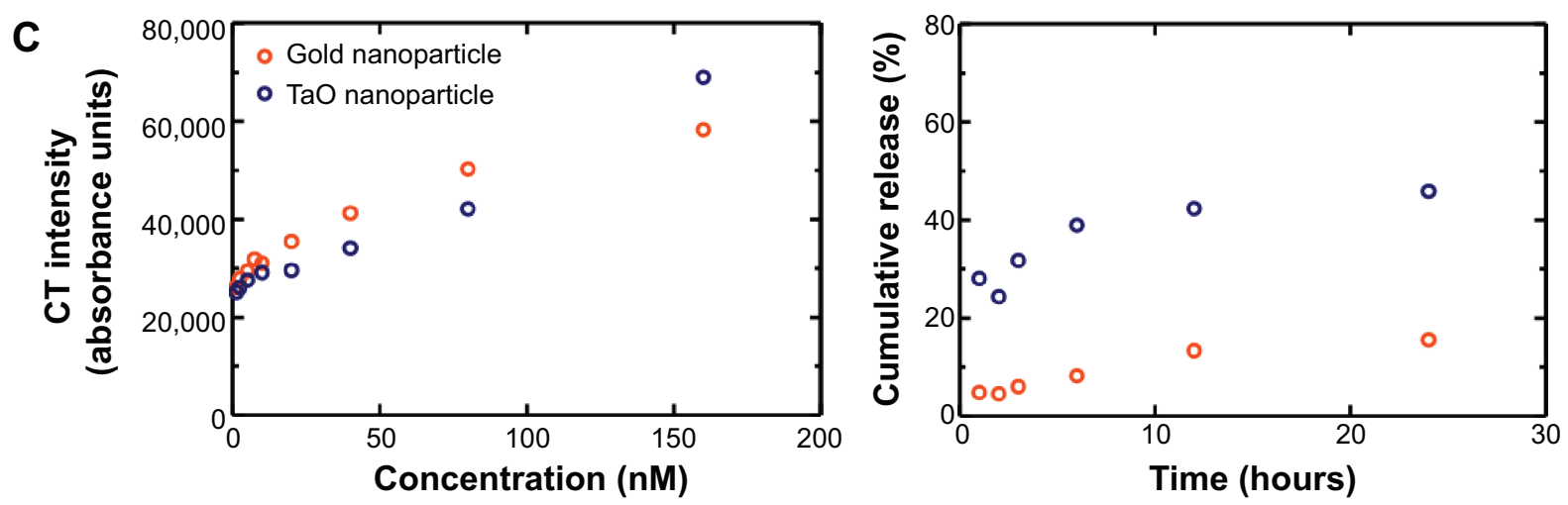

Figure 4 Hydrogels carrying CT contrast nanoparticles were maintained in phosphate-buffered saline for predetermined periods of time (A). CT signals measured in standards and samples collected from wells with hydrogels (B). The cumulative release of particles is calculated based on a standard curve constructed by measuring CT intensities of different concentrations of standard for each nanoparticle (C).

Abbreviations: PBS, phosphate-buffered saline; CT, computed tomography; $\mathrm{TaO}$, tantalum oxide.

proteolysis. Collagen hydrogels are degraded with time under physiological conditions, while the degradation profile depends on the cross-linking conditions of hydrogels. ${ }^{28-33} \mathrm{In}$ general, the greater the extent of cross-linking of hydrogels, the slower their degradation. It is likely that the extent of cross-linking of collagen hydrogels increases with prolonged cross-linking time, resulting in slower in vitro degradation and subsequent release of biomolecules.

TGF- $\beta 1$ induces the proliferation of gingival fibroblasts and their differentiation into myofibroblasts in the gingiva. ${ }^{10}$ In this study, the function of TGF- $\beta 1$ in facilitating the recovery and proliferation of human gingival cells was assessed when TGF- $\beta 1$ was incorporated into and excreted from physiologically degraded collagen hydrogel (Figure 5). Based on the release profile in Figures 2 and 3, it was anticipated that TGF- $\beta 1$ would exhibit a similar release profile from the hydrogel. TGF- $\beta 1$ and other imaging nanoparticles (eg, gold, ferritin, or $\mathrm{TaO}$ nanoparticles) were comixed in the hydrogel and the release profiles were measured. The enzyme-linked immunosorbent assay result (see Supplementary materials Figures S1-3) also confirmed the release of TGF- $\beta 1$ from the hydrogel interacting with gingival cells during coculture. Scratch wounds were inflicted on human gingival cells and the surface areas of the wounds that were generated did not differ between groups: $500 \pm 20 \mathrm{~mm}^{2}, 400 \pm 50 \mathrm{~mm}^{2}$, and $450 \pm 30 \mathrm{~mm}^{2}$ for cells exposed to vehicle control, $10.1 \mu \mathrm{M}$ of free TGF- $\beta 1$, and TGF- $\beta 1$-incorporating collagen hydrogel, respectively. Cells from all groups repopulated the denuded areas within 24 hours. However, the kinetics of the process between cells treated with or without TGF- $\beta 1$ were different 
A
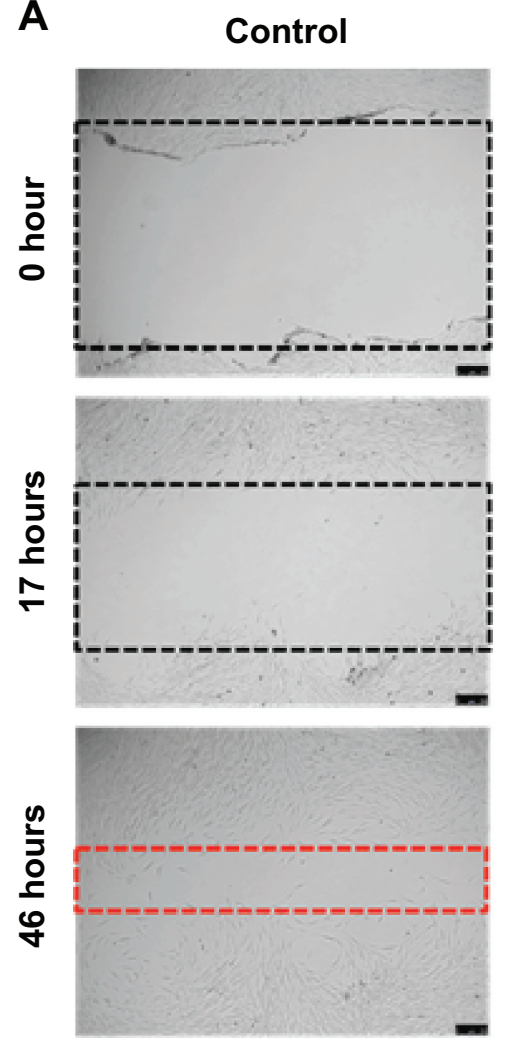

TGF- $\beta 1$
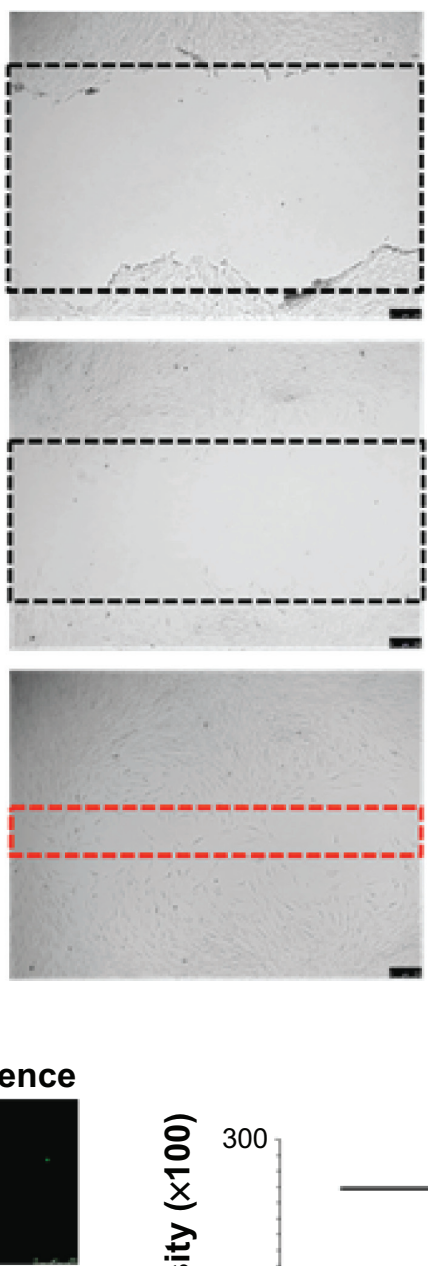

B

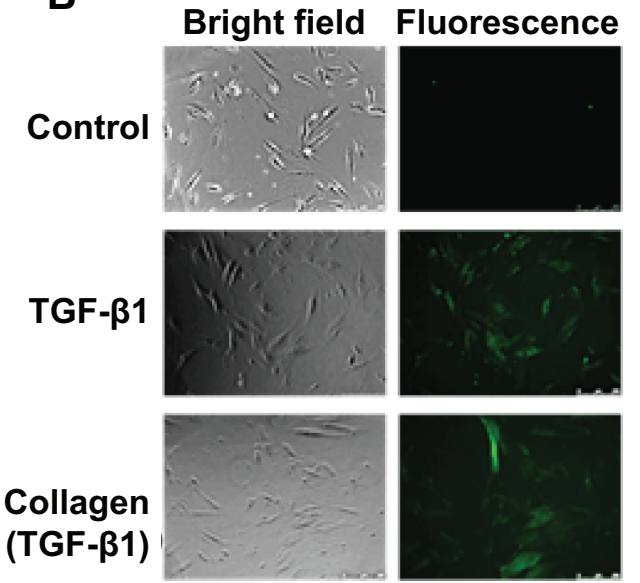

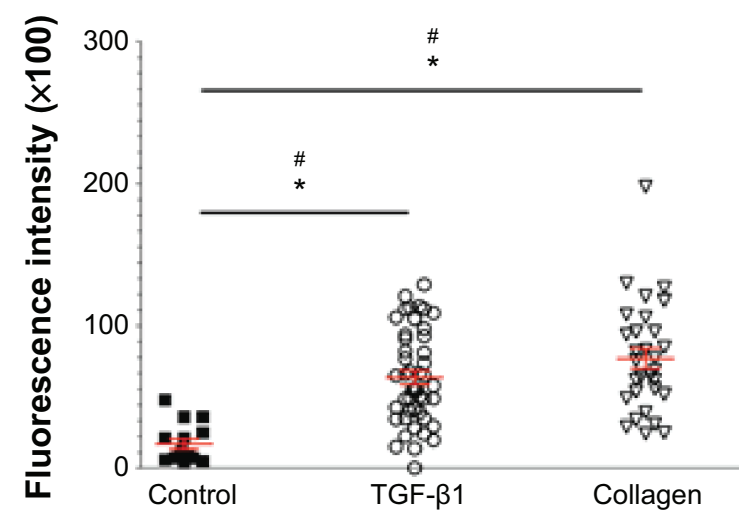

Figure 5 Evaluation of hydrogel efficiency for promoting the recovery of human gingiva.

Notes: Neat TGF- $\beta$ I and TGF- $\beta$ I-carrying hydrogels incubated with human gingival cells display more rapid shrinkage of damaged areas than the control (A). Proliferation of human gingival cells obtained in samples cultured with neat TGF- $\beta$ I and TGF- $\beta$ I carrying hydrogels. The number and metabolic activity of live cells are predominant in samples cultured with neat TGF- $\beta$ I and TGF- $\beta$ I-carrying hydrogels (B). Statistical significance is calculated by comparing results for the control and for each sample. Mean fluorescent intensity, ${ }^{*} P<0.05$, one-tailed Mann-Whitney $U$-test; number of responses, ${ }^{\# P}<0.05$, chi-square with Yate's correction. Data are representative of three experiments. Abbreviation: TGF- $\beta$ I, transforming growth factor-betal.

(Figure 5). Moreover, the cells treated with collagen hydrogels showed a recovery rate similar to that of cells incubated with free TGF- $\beta 1$.

To assess whether TGF- $\beta 1$ changed the contribution of cell proliferation to wound repair, human gingival cell monolayers were tested for incorporation of Calcein-AM 24 hours after scratch injury (Figure 5). The number and metabolic activity of live cells was statistically significant in samples cultured with free TGF- $\beta 1$ and TGF $\beta 1$-carrying hydrogels compared with the control. This indicates that TGF- $\beta 1$ released from the collagen hydrogel has the equal efficacy comparing with free TGF- $\beta 1$.

Since our system was designed to let the collagen hydrogels physically interact with cells, it is important to consider the possibility of surface effects that may modulate the biological behavior of gingival fibroblasts. 
In a recent study, human gingival fibroblasts grown on a rough titanium disk surface displayed proliferation and cell differentiation. ${ }^{34}$ This suggests that additional processing of the surface of collagen hydrogels would help them be favorable for cells to become attached and grow well. These surface effects were also observed in other studies showing, osteoblasts the etched surface of titanium promoted high Co I production and increased level of $\alpha 2-\beta 1$ receptor. ${ }^{35-37}$ In this regard, future work should be designed to introduce optimization of surface roughness in hydrogels to create a favorable microenvironment for gingival cells.

The successful delivery and suitable physiochemical interactions with cells are benefits of the hydrogels for their in vivo application. However it should be noted that our system cannot carry hydrophobic drug molecules within collagen matrices. Therefore, hydrophilic surface termination of delivering drugs must be taken care of. In vivo efficacy and safety tests of our collagen hydrogel are also required before any further consideration for potential clinical applications.

\section{Conclusion}

The collagen hydrogels that we developed for the sustained release of biomolecular particles may be suitable for use as delivery systems for many kinds of bioactive proteins and drugs. Further studies are currently being pursued to evaluate the biocompatibility of our collagen hydrogel system ${ }^{38}$ and modulation of hydrogels for delivery of biomolecules and particles.

\section{Acknowledgments}

We thank Dr Jiadi Xe at the Johns Hopkins University School of Medicine for his assistance with the CT imaging. This work is supported by KIST (Institutional Project 2E24680), a National Research Foundation of Korea grant by the Korean government (MEST; 2008-0061856), and the Basic Science Research Program through the National Research Foundation of Korea funded by MEST (2013R1A1A1012653).

\section{Disclosure}

The authors report no conflicts of interest in this work.

\section{References}

1. Marcopoulou CE, Vavouraki HN, Dereka XE, Vrotsos IA. Proliferative effect of growth factors TGF-beta1, PDGF-BB and rhBMP-2 on human gingival fibroblasts and periodontal ligament cells. $J$ Int Acad Periodontol. 2003;5(3):63-70.

2. Hong HH, Uzel MI, Duan C, Sheff MC, Trackman PC. Regulation of lysyl oxidase, collagen, and connective tissue growth factor by TGF-beta 1 and detection in human gingiva. Lab Invest. 1999;79(12):1655-1667.
3. Sloan AJ, Lynch CD. Dental tissue repair: novel models for tissue regeneration strategies. Open Dent J. 2012;6:214-219.

4. Dobie K, Smith G, Sloan AJ, Smith AJ. Effects of alginate hydrogels and TGF-beta 1 on human dental pulp repair in vitro. Connect Tissue Res. 2002;43(2-3):387-390.

5. Polverini PJ. Cellular adhesion molecules. Newly identified mediators of angiogenesis. Am J Pathol. 1996;148(4):1023-1029.

6. Thompson JA, Anderson KD, DiPietro JM, et al. Site-directed neovessel formation in vivo. Science. 1988;241(4871):1349-1352.

7. Holland TA, Tabata Y, Mikos AG. Dual growth factor delivery from degradable oligo(poly(ethylene glycol) fumarate) hydrogel scaffolds for cartilage tissue engineering. J Control Release. 2005;101(1-3):111-125.

8. Friess W. Collagen - biomaterial for drug delivery. Eur JPharm Biopharm. 1998;45(2):113-136.

9. Tipton DA, Howell KJ, Dabbous MK. Increased proliferation, collagen, and fibronectin production by hereditary gingival fibromatosis fibroblasts. J Periodontol. 1997;68(6):524-530.

10. Montesano R, Orci L. Transforming growth factor beta stimulates collagen-matrix contraction by fibroblasts: implications for wound healing. Proc Natl Acad Sci U S A. 1988;85(13):4894-4897.

11. Zagai U, Fredriksson K, Rennard SI, Lundahl J, Sköld CM. Platelets stimulate fibroblast-mediated contraction of collagen gels. Respir Res. 2003;4:13.

12. Raeber GP, Lutolf MP, Hubbell JA. Molecularly engineered PEG hydrogels: a novel model system for proteolytically mediated cell migration. Biophys J. 2005;89(2):1374-1388.

13. Silver FH, Pins G. Cell growth on collagen: a review of tissue engineering using scaffolds containing extracellular matrix. $J$ Long Term Eff Med Implants. 1992;2(1):67-80.

14. Lee J, Cuddihy MJ, Kotov NA. Three-dimensional cell culture matrices: state of the art. Tissue Eng Part B Rev. 2008;14(1):61-86.

15. McCall JD, Lin CC, Anseth KS. Affinity peptides protect transforming growth factor beta during encapsulation in poly(ethylene glycol) hydrogels. Biomacromolecules. 2011;12(4):1051-1057.

16. Gilbert DL, Teruo O, Teruo M, Sung Wan K. Macromolecular diffusion through collagen membranes. Int J Pharm. 1988;47(1-3):79-88.

17. Wallace DG, Rosenblatt J. Collagen gel systems for sustained delivery and tissue engineering. Adv Drug Deliv Rev. 2003;55(12):1631-1649.

18. Maeda M, Tani S, Sano A, Fujioka K. Microstructure and release characteristics of the minipellet, a collagen-based drug delivery system for controlled release of protein drugs. J Control Release. 1999;62(3): 313-324.

19. Kandamchira A, Selvam S, Marimuthu N, Janardhanan SK, Fathima NN. Influence of functionalized nanoparticles on conformational stability of type I collagen for possible biomedical applications. Mater Sci Eng C Mater Biol Appl. 2013;33(8):4985-4988.

20. Bao G, Mitragotri S, Tong S. Multifunctional nanoparticles for drug delivery and molecular imaging. Аnпи Rev Biomed Eng. 2013; 15:253-282.

21. Lee JH, Kim JW, Cheon J. Magnetic nanoparticles for multi-imaging and drug delivery. Mol Cells. 2013;35(4):274-284.

22. Shilo M, Reuveni T, Motiei M, Popovtzer R. Nanoparticles as computed tomography contrast agents: current status and future perspectives. Nanomedicine (Lond). 2012;7(2):257-269.

23. Rajan N, Habermehl J, Coté MF, Doillon CJ, Mantovani D. Preparation of ready-to-use, storable and reconstituted type I collagen from rat tail tendon for tissue engineering applications. Nat Protoc. 2006;1(6):2753-2758.

24. Hwang MP, Lee JW, Lee KE, Lee KH. Think modular: a simple apoferritin-based platform for the multifaceted detection of pancreatic cancer. ACS Nano. 2013;7(9):8167-8174.

25. Choi J, Park S, Stojanović Z, et al. Facile solvothermal preparation of monodisperse gold nanoparticles and their engineered assembly of ferritin-gold nanoclusters. Langmuir. 2013;29(50):15698-15703.

26. Oh MH, Lee N, Kim H, et al. Large-scale synthesis of bioinert tantalum oxide nanoparticles for X-ray computed tomography imaging and bimodal image-guided sentinel lymph node mapping. J Am Chem Soc. 2011;133(14):5508-5515. 
27. Ko K, Arora P, Lee W, McCulloch C. Biochemical and functional characterization of intercellular adhesion and gap junctions in fibroblasts. Am J Physiol Cell Physiol. 2000;279(1):C147-C157.

28. Drury JL, Mooney DJ. Hydrogels for tissue engineering: scaffold design variables and applications. Biomaterials. 2003;24(24):4337-4351.

29. Singh MP, Stefko J, Lumpkin JA, Rosenblatt J. The effect of electrostatic charge interactions on release rates of gentamicin from collagen matrices. Pharm Res. 1995;12(8):1205-1210.

30. Wang AY, Foss CA, Leong S, Mo X, Pomper MG, Yu SM. Spatiotemporal modification of collagen scaffolds mediated by triple helical propensity. Biomacromolecules. 2008;9(7):1755-1763.

31. Popovtzer R, Agrawal A, Kotov NA, et al. Targeted gold nanoparticles enable molecular CT imaging of cancer. Nano Lett. 2008;8(12): 4593-4596.

32. Reuveni T, Motiei M, Romman Z, Popovtzer A, Popovtzer R. Targeted gold nanoparticles enable molecular CT imaging of cancer: an in vivo study. Int J Nanomedicine. 2011;6:2859-2864.

33. Tabata Y, Miyao M, Ozeki M, Ikada Y. Controlled release of vascular endothelial growth factor by use of collagen hydrogels. J Biomater Sci Polym Ed. 2000;11(9):915-930.
34. Ramaglia L, Capece G, Di Spigna G, Bruno MP, Buonocore N, Postiglione L. Effects of titanium surface topography on morphology and in vitro activity of human gingival fibroblasts. Minerva Stomatol. 2013;62(7-8):267-280.

35. Ramaglia L, Postiglione L, Di Spigna G, Capece G, Salzano S, Rossi G. Sandblasted-acid-etched titanium surface influences in vitro the biological behavior of SaOS-2 human osteoblast-like cells. Dent Mater J. 2011;30(2):183-192.

36. Postiglione L, Di Domenico G, Ramaglia L, di Lauro AE, Di Meglio F, Montagnani S. Different titanium surfaces modulate the bone phenotype of SaOS-2 osteoblast-like cells. Eur J Histochem. 2004;48(3):213-222.

37. Postiglione L, Di Domenico G, Ramaglia L, et al. Behavior of SaOS-2 cells cultured on different titanium surfaces. J Dent Res. 2003; 82(9):692-696.

38. Jang GH, Hwang MP, Kim SY, Jang HS, Lee KH. A systematic in-vivo toxicity evaluation of nanophosphor particles via zebrafish models. Biomaterials. 2014;35(1):440-449. 


\section{Supplementary materials}

A

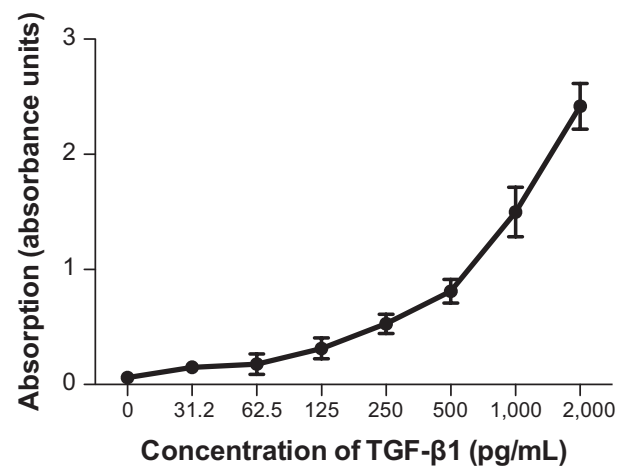

B

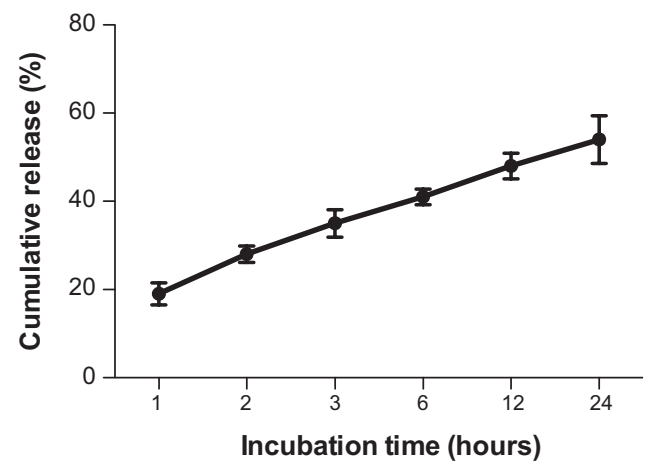

Figure SI The release profile of TGF- $\beta$ I incorporated in collagen hydrogel.

Notes: The standard curve of absorption from the dilution series of TGF- $\beta$ I was constructed (A) and the actual release of TGF- $\beta$ I is back-calculated based on the standard (B). Over 24 hours of incubation, the average amount of TGF- $\beta$ I accumulated in the buffer was around $52 \%$. Each experiment was performed in triplicate. Abbreviation: TGF- $\beta$ I, transforming growth factor-betal.
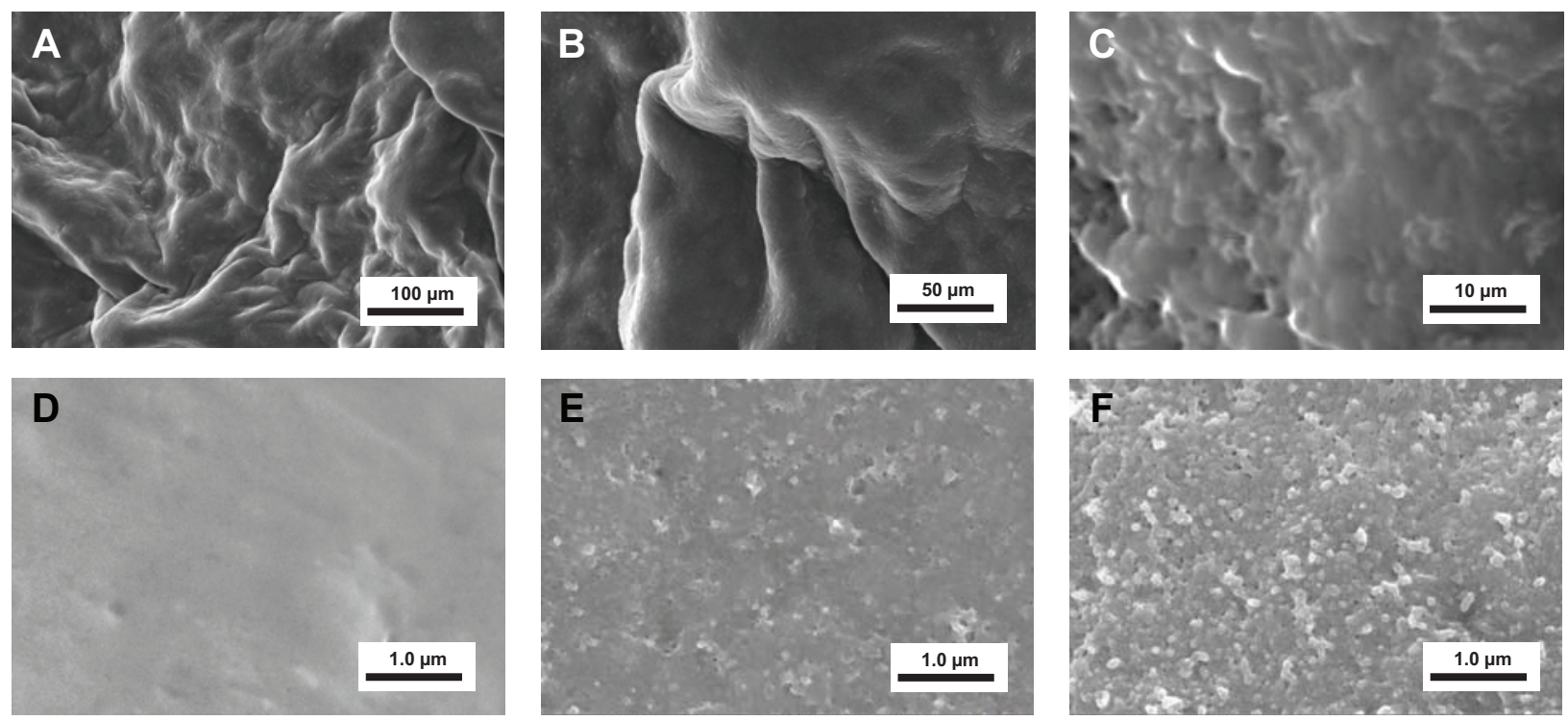

Figure S2 Scanning electron microscopy analysis of collagen hydrogels.

Notes: Porous, microscopic structures of hydrogels were observed (A-C). Comparing the morphologic surface variations between plain (D) and TGF- $\beta$ I-modified (E) $(100 \mathrm{pg} / \mathrm{mL})$ and $(\mathbf{F})(1,000 \mathrm{pg} / \mathrm{mL})$ collagen hydrogels. 


\section{Optimizing collagen hydrogel preparation}

In order to optimize the hydrogel for sustained release of biomolecules and nanoparticles, we have modulated various parameters (eg, concentration, temperature, curing duration, $\mathrm{pH}$ ) to produce the cone-shaped collagen hydrogel. Here we list several parameters tested and the resulting hydrogels.

\begin{tabular}{|c|c|c|}
\hline Parameters & Variations & Results/effects \\
\hline \multirow[t]{3}{*}{ Temperature } & $37^{\circ} \mathrm{C}$ & Well cured for $1.5 \%$ collagen \\
\hline & $4^{\circ} \mathrm{C}$ & Difficult to solidify \\
\hline & Room temperature & Difficult to solidify \\
\hline \multirow[t]{3}{*}{$\mathrm{pH}$} & $\mathrm{pH} 5-6$ & Difficult to solidify, large pore size $(\sim 200 \mu \mathrm{m})$ \\
\hline & $\mathrm{pH} 6-7$ & Weakly cured, medium pore size $(\sim 120 \mu \mathrm{m})$ \\
\hline & $\mathrm{pH} 7-8$ & Well cured, small pore size $(\sim 80 \mu \mathrm{m})$ \\
\hline \multirow[t]{4}{*}{ Collagen stock concentration } & $1.0 \%(w / v)$ & Gelation takes a long time ( $\sim 8$ hours), relatively large pore size $(\sim 200 \mu \mathrm{m})$ \\
\hline & $1.5 \%(w / v)$ & Relatively large pore size $(\sim 200 \mu \mathrm{m})$ \\
\hline & $2.0 \%(w / v)$ & Nonhomogenous gelation, relatively small pore size $(\sim 100 \mu \mathrm{m})$ \\
\hline & $2.5 \%(w / v)$ & Nonhomogeneous gelation, relatively small pore size $(\sim 100 \mu \mathrm{m})$ \\
\hline \multirow[t]{3}{*}{ Curing duration } & $\sim 30$ minutes & Less cured for I.5\% collagen \\
\hline & $\sim 60$ minutes & Well cured for $1.5 \%$ collagen \\
\hline & $\sim 90$ minutes & Well cured for $1.5 \%$ collagen, susceptible to drying of hydrogel \\
\hline
\end{tabular}

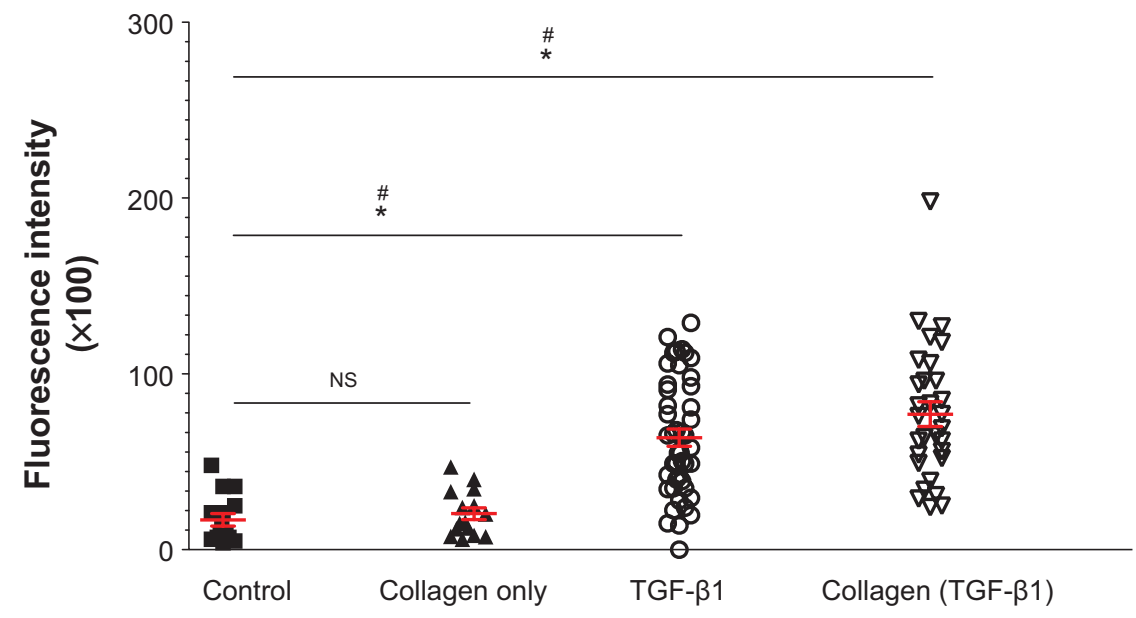

Figure S3 The frequency and activity of living cells are increased in samples incubated with either neat TGF- $\beta$ I or TGF- $\beta$ I-carrying hydrogels.

Notes: Statistical significance was calculated by comparing results for the control, neat collagen, and for each sample. Mean fluorescent intensity, $* P<0.05$, one-tailed Mann-Whitney $U$-test; number of responses: ${ }^{\#} P<0.05$, chi-square with Yate's correction. NS denotes not statistically significant in the statistical test. Data are representative of three experiments.

Abbreviation: TGF- $\beta$ I, transforming growth factor-betal.

\section{Publish your work in this journal}

The International Journal of Nanomedicine is an international, peerreviewed journal focusing on the application of nanotechnology in diagnostics, therapeutics, and drug delivery systems throughout the biomedical field. This journal is indexed on PubMed Central, MedLine, CAS, SciSearch $\AA$, Current Contents ${ }^{\circledR} /$ Clinical Medicine,
Journal Citation Reports/Science Edition, EMBase, Scopus and the Elsevier Bibliographic databases. The manuscript management system is completely online and includes a very quick and fair peer-review system, which is all easy to use. Visit http://www.dovepress.com/ testimonials.php to read real quotes from published authors. 